\title{
Weather Factor Effect with Distance at 5 G Candidate Frequencies in Mosul.
}

\author{
Younis M. Abbosh, Zaid A. Sideeq \\ younisabb@yahoo.com,zaideyad@yahoo.com \\ Department of communication engineering, Ninevah University, Iraq
}

\begin{abstract}
This paper presents the effect of rain rate, humidity, and temperature at multiple frequencies in the millimeter-wave band in Mosul city. The fifth-generation has several candidate frequencies above $10 \mathrm{GHz}$, such as $28,37,60$, and $73 \mathrm{GHz}$. The results show that the attenuation due to weather factors does not represent a problem for short distances but Losses increase with frequency, and at a large distance $(1 \mathrm{~km})$, they become very large.
\end{abstract}

Keywords: 5G, channel model, NYUSim

\section{Introduction}

Humanity witnessed rapid growth and development in mobile wireless communication in the last few decades. This evolution consists of generations $(\mathrm{G})$ and foreseen generations are still coming in the future. Mobile wireless generations started with first-generation $(1 G)$ then followed by $2 \mathrm{G}, 3 \mathrm{G}, 4 \mathrm{G}$ and then the upcoming $5 \mathrm{G}$ which is still under research. These generations differ from each other incapacity, data rate, services, latency, technologies, and even challenges. [1]

\section{Fifth-generation}

The demand for higher mobile data rates increases year after year in a dramatic manner especially in the last few years, which pushed existing mobile technology to its limits according to the Cisco report.[2]

The fifth-generation is still under study but it will give the user a revolutionary experience that he did not go through it before.[1]

The fifth-generation is expected to have great and important changes not only in the term of bandwidth but it will change the fundamental purpose of cell phones. The user is going to experience something like never before. The $5 \mathrm{G}$ will support worldwide wireless web (WWWW).[3]

The work on $5 \mathrm{G}$ requirements, which is divided into two stages, is done by the different organizations. The first stage concentrates on the $5 \mathrm{G}$ vision, which includes use cases and high abilities of the 5G network.[4] In this stage, ITU released its vision testament and determined the abilities of 5G in 2015.[5] The third-generation partner project (3GPP) also searched use cases and abilities of 5G in 2015. Next Generation Mobile Networks (NGMN) identified more use cases and abilities and published it in a white paper in the same year.[6]In the second stage, 
the attention was on high detailed deployment scenarios and requirements of each scenario. The most remarkable report in stage 2 is IMT-2020, which is introduced by the International Telecommunication Union-Radio communication Sector (ITU-R).[7]

\section{Keys of 5G}

Ten features play important role and have the main contribution to the emergence of the fifth generation of mobile systems, these are some of them:

\subsection{Various radio access technology types}

5G will not use a specific type of radio access technology (RAT) but it will use different types of RAT starting from using enhanced versions of previous generations' RATs in addition to new RATs.[8]

\subsection{Hyperdense small-cell deployment}

In order to meet the high increase in mobile data traffic cell sizes are made smaller which also has leverage on energy and spectral efficiency. This technique is also called heterogeneous Networks (HetNet).[8], [9]

\subsection{New backhaul links}

5G relays on small cells densification to achieve 1000X capacity, therefore; backhaul link needs to be able to carry the tremendous data traffic. The motivation of backhaul redesigning is the very high cost of using fiber cables to make a connection between base stations (BS) and cellular networks.[2]

\subsection{Machine Type Communication (MTC)}

It is another important feature of $5 \mathrm{G}$, which provides a connection between large numbers of devices.[10]

\subsection{Millimeter-Wave RATs}

The rapid growth of the number of devices and mobile data traffic in indoor and outdoor scenarios, huge unlicensed bandwidth available in the millimeter-wave band and the small wavelength open the way to build small antennas and massive antenna arrays. Massive MIMO enables pencil-beam forming to achieve high spatial spectrum reuse and signal-tointerference ratios.[10], [11]

\subsection{New spectrum allocation}

Mobile data traffic and the number of connections are expected to increase in an exponential manner after 2020.[4]The current spectrum shortage becomes more obvious with the increasing demand for larger capacity. The current bandwidths below $6 \mathrm{GHz}$ are not satisfying $5 \mathrm{G}$ requirements while the millimeter wave band has abundant bandwidth making 
its major part of 5G. Current researches interested in $28 \mathrm{GHz}$ band, $38 \mathrm{GHz}$ band, $60 \mathrm{GHz}$ band and E-band (71-76 GHz and 81-86 GHz).[12]

\section{Losses at millimeter waves}

The stakeholders are paying attention to the frequencies in the region between 30-300 $\mathrm{GHz}$ due to the large bandwidth which is available in this region. The signals at frequencies above $30 \mathrm{GHz}$ cannot propagate for many miles as those below $30 \mathrm{GHz}$. In the millimeter-wave bands, factors that were neglected at low frequencies are taken into the account.

\subsection{Free space loss.}

Loss, frequency, and distance can be related in a mathematical expression which is shown in equation 1. It is assumed that the transmitter $(\mathrm{Tx})$ and receiver $(\mathrm{Rx})$ have isotropic antennas.

$L_{F S L}=\left(\frac{4 \pi d}{\lambda}\right)^{2}$

Where LFSL is the free-space loss, $\mathrm{d}$ is the distance between transmitter and receiver, and $\lambda$ is the wavelength.[13]

\subsection{Atmospheric losses.}

Besides free space loss, there are raindrops and oxygen's molecules that can cause additional losses. Some frequencies suffer larger attenuation than others do because of the mechanical resonant frequencies of the gas molecules.

\subsubsection{Rain.}

Raindrops are not alike in the term of size and not quite near radio wavelength's size. Figure 1 shows attention caused by different rain rates. [13] 


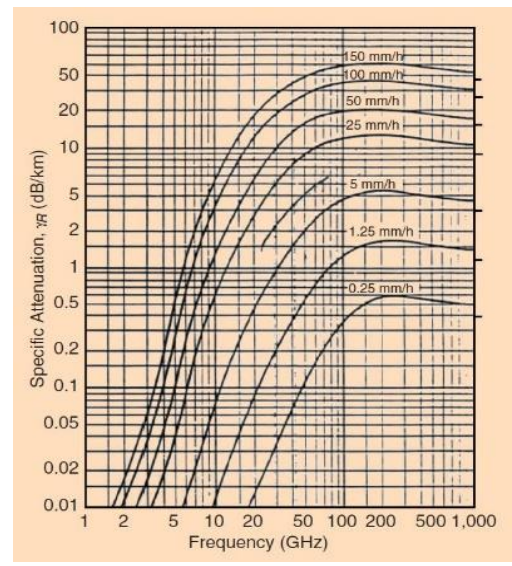

Fig.1: Losses due to rain at millimeter-wave band.[13]

\subsubsection{Atmospheric Gaseous.}

When the signal travels through the atmosphere, it suffers from attenuation due to oxygen, water vapor, and molecules of other gases that absorb signal energy.

\subsection{Scattering/ Diffraction.}

A signal transmitted by the transmitter can reach the receiver even if the line of sight between Tx and Rx is absent because a signal gets reflected or diffracted from objects that are near the receiver. The millimeter-wave signals have short wavelengths that lead to low diffraction.[13]

\section{Simulation and results}

The researchers at New York University had developed a simulator based on data collected in New York Cityand Daejeon, Korea for urban macro and urban micro. The simulator is called NYUSim. NYUSim is a statistical spatial channel model.[14]The results of the simulation in this paper are taken from NYUSim to study the weather factor effect on $5 \mathrm{G}$ in Mosul city. Weather parameters such as temperature, humidity, and rain rate have been taken from the Iraqi meteorological organization and seismology of Mosul station for the period 1988 to 2007 .

\subsection{Loss vs distance at $28 \mathrm{GHz}$.}

A simulation had been made for a 4X4 MIMO system, where antenna consists of a 4X1 uniform linear array (ULA) of HPBW $23.8^{\circ}$ in E-plane and 44.7 in H-plane with $0.5 \lambda$ spacing between elements.[15]. The signal is transmitted from the base station at power $30 \mathrm{dBm}$ in the urban microcell scenario. The simulation made in months February and August at distances 10, 100 and $1000 \mathrm{~m}$. The results of the simulation are plotted in Figure 2. 


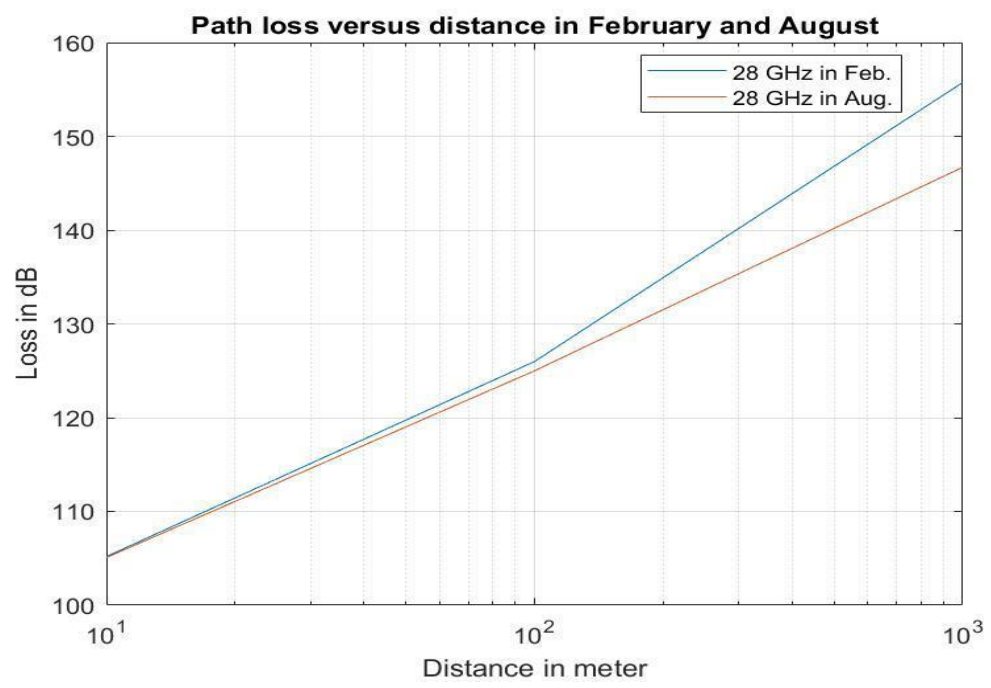

Fig. 2. path loss of $28 \mathrm{GHz}$ with a distance.

\subsection{Loss vs distance at $37 \mathrm{GHz}$.}

In the same way of the $28 \mathrm{GHz}$ simulation, a simulation had been made to show path loss of $37 \mathrm{GHz}$ signal at different distances. The simulation parameters like transmit power, and scenario of interest is not changed. The antenna consists of 5 elements with HPBW $15.2^{\circ}$ in Eplane and $45^{\circ}$ in $\mathrm{H}$-plane at both transmitter and receiver with $0.6 \lambda$ spacing between elements.[16]. The simulation also made in February and August and the results of simulation gathered and plotted in Figure 3.

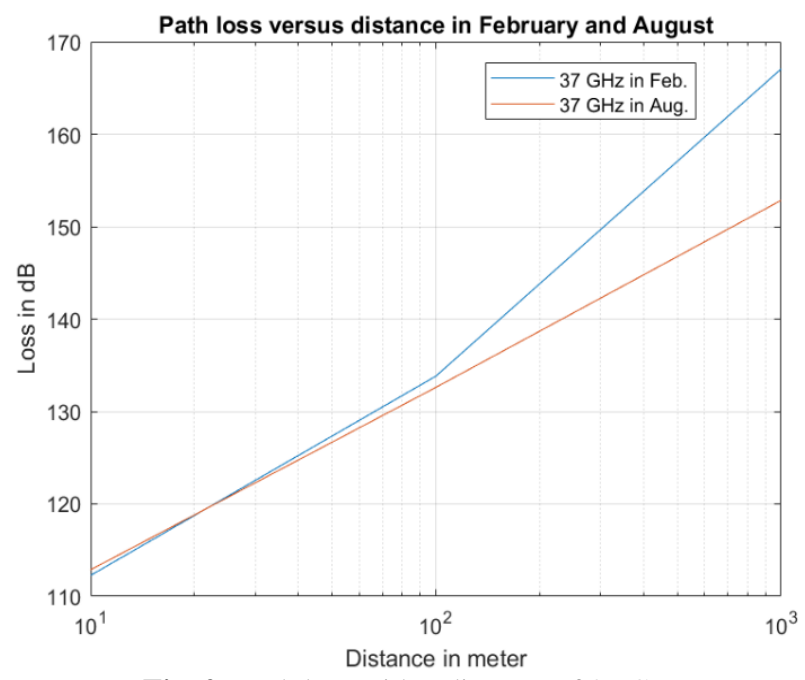

Fig. 2. path loss with a distance of $37 \mathrm{GHz}$. 


\subsection{Loss vs distance at $60 \mathrm{GHz}$.}

To see how loss changes with distance at $60 \mathrm{GHz}$, the following simulation parameters utilized: 8 elements antenna array at $\mathrm{Tx}$ and $\mathrm{Rx}$ with $50^{\circ}$ and $20^{\circ} \mathrm{HPBW}$ in $\mathrm{E}$ and $\mathrm{H}$ plane respectively with $0.5 \lambda$ spacing between elements.[17], [18]The simulation made for February and August weather conditions and the results of simulation is plotted in Figure 4.

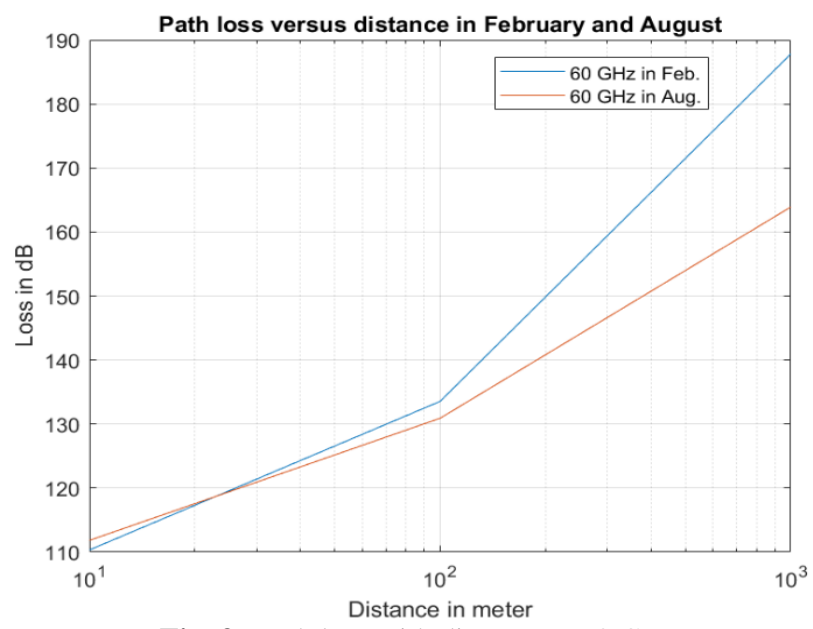

Fig. 3. path loss with distance at $60 \mathrm{GHz}$.

$60 \mathrm{GHz}$ suffers from higher attenuation compared to other frequencies due to oxygen absorption; therefore, it is not preferable for outdoor scenarios. $60 \mathrm{GHz}$ usually used for wireless local area networks (WLAN) for the indoor scenario.[19]

\subsection{Loss vs distance at $73 \mathrm{GHz}$.}

Loss due to weather factors at $73 \mathrm{GHz}$ is calculated in a simulation. The antenna used for 73 $\mathrm{GHz}$ is horn antenna of beam width $10^{\circ}$ in E-plane and $10^{\circ}$ in H-plane.[20] The transmitter power and scenario are the same as the previous simulations. Weather factors of February and August were used. Figure 5 shows the results of the simulation. 


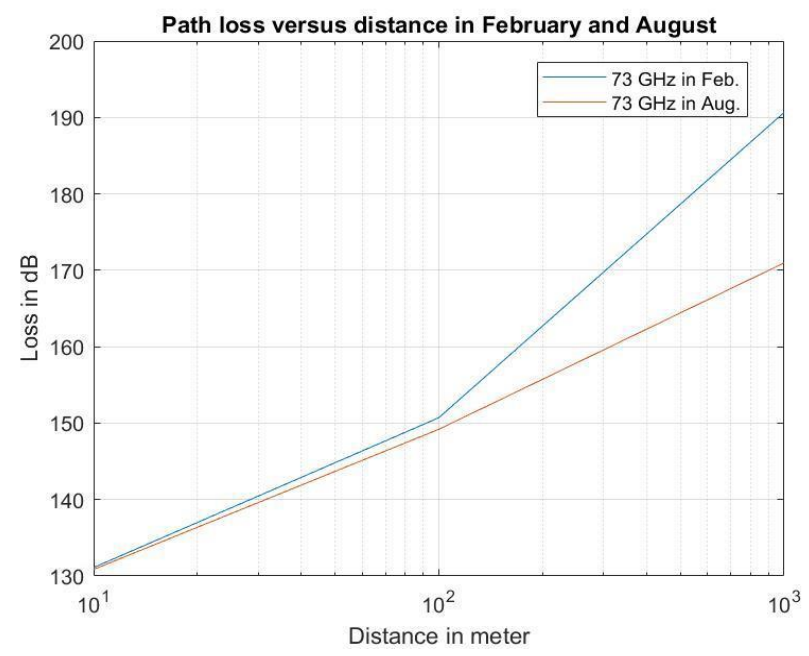

Fig. 4. loss with a distance of $73 \mathrm{GHz}$.

\section{Conclusions}

The attenuation due to weather factors does not represent a problem for short distances. The rain has the largest impact on the signal power level and its effect increases with rain rate and distance. The loss difference between winter and summer for $28 \mathrm{GHz}$ is about $1 \mathrm{~dB}$ in Mosul. The loss difference between winter and summer is about $2 \mathrm{~dB}$ for $37 \mathrm{GHz}$, about $4 \mathrm{~dB}$ for $60 \mathrm{GHz}$ and about $2 \mathrm{~dB}$ for $73 \mathrm{GHz}$. Losses increase with frequency and at a large distance like $1 \mathrm{~km}$, they become very large.

\section{References:}

[1]L. J. Vora, "Evolution of mobile generation technology: $1 \mathrm{G}$ to $5 \mathrm{G}$ review of upcoming wireless technology 5G,” Int. J. Mod. Trends Eng. Res., vol. 2, no. 10, pp. 281-291, 2015.

[2]R. Vannithamby and S. Talwar, Eds., Towards 5G: Applications, Requirements and Candidate Technologies, 1st ed. Chichester, UK: John Wiley \& Sons, 2017.

[3]M. G. Kachhavay and A. P. Thakare, "5G Technology-Evolution and Revolution,” Int. J. Comput. Sci. Mob. Comput., vol. 3, no. 3, p. 8, Mar. 2014.

[4]W. Xiang, Xuemin, and K. Zheng, 5G Mobile Communications. NY: Springer Science+Business Media, 2016.

[5]Recommendation ITU-R M.2083-0, "IMT Vision - Framework and overall objectives of the future development of IMT for 2020 and beyond," international telecommunication union, 2015.

[6]N. Alliance, "NGMN 5G White Paper," Next generation mobile networks, Final Deliverable, 2015. [7]ITU-R, "Workplan, timeline, process and deliverables for the future development of IMT," vol. 19, no. November, 2012.

[8]J. Rodriguez, Fundamentals of 5G Mobile Networks, 1st ed. John Wiley \& Sons, 2015.

[9]Y. Yang, J. Xu, G. Shi, and C.-X. Wang, 5G Wireless Systems, 1st ed. Cham: Springer International Publishing, 2018.

[10]A. Osseiran, P. Marsch, and J. F. Monserrat, Eds., 5G mobile and wireless communications technology, 1st ed. United Kingdom : New York: Cambridge University Press, 2016. 
[11]V. Degli-Espostiet al., "Ray-Tracing-Based mm-Wave Beamforming Assessment,” IEEE Access, vol. 2, pp. 1314-1325, 2014.

[12]Y. Niu, Y. Li, D. Jin, L. Su, and A. V. Vasilakos, "A Survey of Millimeter Wave (mmWave) Communications for 5G: Opportunities and Challenges," arXiv, vol. 21, no. 8, pp. 2657-2676, Feb. 2015.

[13]M. Michael and B. Pattan, "Millimeter wave propagation: spectrum managment implications," IEEE microwave magazine, no. 70, 2005.

[14]T. S. Rappaport, S. Sun, and M. Shafi, "Investigation and Comparison of 3GPP and NYUSIM Channel Models for 5G Wireless Communications," in IEEE Vehicular Technology Conference, Toronto, Canada, 2017.

[15]M. H. A. Saada, "Design of Efficient Millimeter Wave Planar Antennas for 5G Communication Systems.," M.Sc. dissertation, The Islamic University-Gaza, Faculty of Engineering, 2017.

[16]F. Oktafiani and Y. N. Wijayanto, "Analysis of printed patch antenna array for $37 \mathrm{GHz}$ point-topoint wireless links," in 2016 22nd Asia-Pacific Conference on Communications (APCC), pp. 379382,Indonesia, 2016,.

[17]Z. N. Chen, X. Qing, M. Sun, K. Gong, and W. Hong, “60-GHz antennas on PCB," in The 8th European Conference on Antennas and Propagation (EuCAP 2014),pp. 533-536, The Hague, Netherlands, 2014

[18]J. Zhan, J. Wen, L. Sun, and X. Shu, "Design of $60 \mathrm{GHz}$ mm-wave patch antenna arrays," in IEEE 16th International Conference on Communication Technology (ICCT), pp. 262-265, China, 2015,

[19]T. S. Rappaport, Y. Xing, J. MacCartney, A. F. Molisch, E. Mellios, and J. Zhang, "Overview of Millimeter Wave Communications for Fifth-Generation (5G) Wireless Networks-with a focus on Propagation Models," IEEE Trans. Antennas Propag., vol. 65, no. 12, pp. 6213-6230, Dec. 2017.

[20]M. R. Akdenizet al., "Millimeter Wave Channel Modeling and Cellular Capacity Evaluation," IEEE J. Sel. Areas Commun., vol. 32, no. 6, pp. 1164-1179, Jun. 2014. 